Nancy J. Tarbell and Torunn Yock, Massachusetts General Hospital, Boston, MA; Henry Friedman, Duke University, Durham, NC; William R. Polkinghorn, Memorial Sloan-Kettering Cancer Center, New York, NY; Tianni Zhou, California State University, Long Beach; Patrick Barnes, Stanford University, Stanford, CA; Zhengjia Chen, Emory University, Atlanta, GA; Peter Burger, Johns Hopkins Hospital, Baltimore, MD; Larry Kun, St Jude Research Hospital, Memphis, TN. Published online ahead of print at www.jco.org on July 15, 2013

Presented in part at the 43rd Annual Meeting of The American Society for Therapeutic Radiology and Oncology, November 4-8, 2001, San Francisco, CA.

Authors' disclosures of potential conflicts of interest and author contributions are found at the end of this article.

Clinical trial information: NCT00003573.

Corresponding author: Nancy J. Tarbell, MD, Massachusetts General Hospital, 55 Fruit St, Boston, MA 02114; e-mail: nancy_tarbell@hms.harvard.edu.

(C) 2013 by American Society of Clinical Oncology

0732-183X/13/3123w-2936w/\$20.00

DOI: 10.1200/JCO.2012.43.9984

\title{
High-Risk Medulloblastoma: A Pediatric Oncology Group Randomized Trial of Chemotherapy Before or After Radiation Therapy (POG 9031)
}

Nancy J. Tarbell, Henry Friedman, William R. Polkinghorn, Torunn Yock, Tianni Zhou, Zhengjia Chen, Peter Burger, Patrick Barnes, and Larry Kun

$$
\begin{array}{llllllll}
\text { A } & \text { B } & \text { S } & \text { T } & \text { R } & \text { A } & \text { C } & \text { T }
\end{array}
$$

\section{Purpose}

To compare event-free survival (EFS) in children with high-risk medulloblastoma randomly assigned to receive either chemotherapy before radiation or chemotherapy after radiation.

\section{Patients and Methods}

One hundred twelve patients were randomly assigned to each arm. Criteria used to categorize patients as high risk included M1-4 disease by modified Chang staging classification, T3b/T4 disease, or greater than $1.5 \mathrm{~cm}^{3}$ of residual tumor after surgery. Postoperatively, children with high-risk medulloblastoma were randomly assigned to two arms, either chemotherapy entailing three cycles of cisplatin and etoposide before radiation (chemotherapy first [CT1]) or the same chemotherapy regimen after radiation (radiation therapy first [RT1]). Both groups received consolidation chemotherapy consisting of vincristine and cyclophosphamide.

\section{Results}

The median follow-up time was 6.4 years. Five-year EFS was $66.0 \%$ in the CT1 arm and $70.0 \%$ in the RT1 arm $(P=.54)$, and 5-year overall survival in the two groups was $73.1 \%$ and $76.1 \%$, respectively $(P=.47)$. In the CT1 arm, 40 of the 62 patients with residual disease achieved either complete or partial remission.

\section{Conclusion}

Five-year EFS did not differ significantly whether, after surgery, patients received chemotherapy before or after radiotherapy.

\section{J Clin Oncol 31:2936-2941. (C) 2013 by American Society of Clinical Oncology}

\section{INTRODUCTION}

Medulloblastoma is the most common pediatric brain malignancy, with approximately 540 new patients diagnosed in the United States each year. ${ }^{1,2}$ For the purposes of treatment, patients are divided by age-less than or greater than 3 years of agewith these groups further subdivided into standardrisk and high-risk groups. Patients greater than 3 years of age are stratified into risk groups based on the extent of disease beyond the original tumor site, as summarized in the $\mathrm{M}$ stage of the Chang staging system, with $\mathrm{M} 1$ or greater and residual tumor size after surgery of greater than $1.5 \mathrm{~cm}^{3}$ considered high risk. ${ }^{3}$

Since medulloblastoma was first described in 1925 by Bailey and Cushing, the futility of surgical resection alone has been recognized, and it was only after the use of postsurgical craniospinal irradiation (CSI) in the 1960s that significant survivability was reported. In the 1970s, randomized trials conducted by the Children's Cancer Group and the International Society of Pediatric Oncology demonstrated improved survival for the high-risk patient when incorporating adjuvant chemotherapy, ${ }^{4,5}$ the results of which Packer et $\mathrm{al}^{6}$ improved on by adding cisplatin to lomustine and vincristine. Current event-free survival (EFS) rates for high-risk patients using fulldose CSI with or without the addition of chemotherapy range from $30 \%$ to $74 \% .{ }^{6-11}$ Packer et $\mathrm{al}^{12}$ later established the value of chemotherapy in the standard-risk patient, for whom the dose of CSI could be lowered to $23.4 \mathrm{~Gy}$ in the setting of similar adjuvant chemotherapy without compromising survivability. More recent trials treating standardrisk medulloblastoma using reduced-dose CSI and adjuvant chemotherapy have produced EFS rates of $81 \%$ to $86 \%$. $^{11,13}$

To improve survival for high-risk medulloblastoma, a number of different strategies have been investigated that attempt to combine chemotherapy with radiation therapy in an optimized manner. 


\begin{tabular}{|c|c|c|c|c|c|c|c|}
\hline \multirow[b]{2}{*}{ Demographic or Clinical Characteristic } & \multicolumn{2}{|c|}{$\begin{array}{l}\text { Chemotherapy First } \\
\quad(n=112) \\
\end{array}$} & \multicolumn{2}{|c|}{$\begin{array}{l}\text { Radiotherapy First } \\
(\mathrm{n}=112) \\
\end{array}$} & \multicolumn{2}{|c|}{$\begin{array}{l}\text { All Patients } \\
(N=224)\end{array}$} & \multirow[b]{2}{*}{$P$} \\
\hline & No. & $\%$ & No. & $\%$ & No. & $\%$ & \\
\hline Sex & & & & & & & $.28^{*}$ \\
\hline Male & 62 & 55 & 70 & 62 & 132 & 59 & \\
\hline Female & 50 & 45 & 42 & 38 & 92 & 41 & \\
\hline \multicolumn{8}{|l|}{ M stage } \\
\hline 0 & 58 & 52 & 58 & 52 & 116 & 52 & $.91^{*}$ \\
\hline 1 & 16 & 14 & 13 & 12 & 29 & 13 & \\
\hline 2 & 19 & 17 & 17 & 15 & 36 & 16 & \\
\hline 3 & 15 & 13 & 19 & 17 & 34 & 15 & \\
\hline 4 & 4 & 4 & 5 & 4 & 9 & 4 & \\
\hline T stage & & & & & & & $.80+$ \\
\hline 1 & 1 & 1 & 1 & 1 & 2 & 1 & \\
\hline 2 & 8 & 7 & 5 & 4 & 13 & 6 & \\
\hline 3 & 18 & 16 & 23 & 20 & 41 & 18 & \\
\hline 3а & 2 & 2 & 4 & 4 & 6 & 3 & \\
\hline $3 b$ & 43 & 38 & 47 & 42 & 90 & 40 & \\
\hline 4 & 39 & 35 & 31 & 28 & 70 & 31 & \\
\hline Unknown & 1 & 1 & 1 & 1 & 2 & 1 & \\
\hline T stage/MO, no RD & & & & & & & $.19^{*}$ \\
\hline $3 b$ & 27 & 68 & 26 & 81 & 53 & 74 & \\
\hline 4 & 13 & 32 & 6 & 19 & 19 & 26 & \\
\hline Race & & & & & & & $.72+$ \\
\hline White & 88 & 79 & 83 & 74 & 171 & 76 & \\
\hline Black & 17 & 15 & 17 & 15 & 34 & 15 & \\
\hline Hispanic & 5 & 4 & 7 & 6 & 12 & 5 & \\
\hline Asian & 1 & 1 & 4 & 4 & 5 & 2 & \\
\hline Hawaiian & 1 & 1 & 1 & 1 & 2 & 1 & \\
\hline $\begin{array}{l}\text { Abbreviation: RD, residual disease. } \\
\text { "Pearson's } \chi^{2} \text { test. } \\
\text { tFisher's exact test. }\end{array}$ & & & & & & & \\
\hline
\end{tabular}

One strategy has been to give preirradiation chemotherapy, the results of which have been mixed. ${ }^{7-9}$ The primary objective of the Pediatric Oncology Group (POG) 9031 multi-institutional study was to compare the clinical outcome of treating high-risk medulloblastoma patients with either preirradiation or postirradiation chemotherapy, with both groups receiving identical consolidation chemotherapy. A second objective was to evaluate whether response to preirradiation chemotherapy in patients with residual disease possessed prognostic significance.

\section{PATIENTS AND METHODS}

\section{Patient Population}

For the purposes of this study, patients with medulloblastoma were considered high risk if they had M1-4 disease by modified Chang staging classification, exhibited T3b/T4 disease at time of surgery, or had greater than $1.5 \mathrm{~cm}^{3}$ of residual tumor after surgery. From July 1990 through March 1996, patients with high-risk medulloblastoma were randomly assigned to receive either chemotherapy entailing three cycles of cisplatin and etoposide before radiation or the same chemotherapy regimen after radiation; both groups received consolidation chemotherapy consisting of vincristine and cyclophosphamide. Patients were eligible if they were age 3 years to 21 years at registration.

An enhanced computed tomography (CT) or magnetic resonance imaging (MRI) scan within 72 hours after surgery or between 10 and 21 days after surgery was required. Patients had to be registered within 4 weeks of the initial diagnostic surgical procedure and were not eligible if they had received prior chemotherapy. In addition, patients had to meet the following laboratory criteria: serum creatinine less than $1.7 \mathrm{mg} / \mathrm{dL}$, ALT less than $1.5 \times$ normal, total bilirubin less than $1.5 \mathrm{mg} / \mathrm{dL}$, absolute neutrophil count (ANC) more than $1,500 / \mu \mathrm{L}$, hemoglobin more than $10 \mathrm{~g} / \mathrm{dL}$, and platelets more than 100,000/ $\mu \mathrm{L}$. Informed consent was obtained from every patient enrolled, and each participating institution opened the protocol after obtaining approval from his or her local human investigation committee in compliance with the Declaration of Helsinki.

On central review, five patients were found to be ineligible. There were 112 eligible patients left in the analysis per treatment arm. Patient characteristics are listed in Table 1 . As expected, more males $(n=132)$ than females $(n=$ 92) were accrued to this study.

\section{Random Assignment}

Patients were assigned randomly to one of two treatments (Fig 1). The chemotherapy first (CT1) arm consisted of three cycles of preirradiation chemotherapy followed by radiation therapy starting at week 10 . The radiotherapy first (RT1) arm consisted of radiation therapy followed by three cycles of identical chemotherapy. Both treatment arms were followed with consolidation chemotherapy (Fig 2).

\section{Chemotherapy}

Two phases of chemotherapy were administered. The first phase consisted of three cycles of cisplatin $90 \mathrm{mg} / \mathrm{m}^{2}$ and etoposide $150 \mathrm{mg} / \mathrm{m}^{2}$ administered every 4 weeks intravenously on day 1 of each cycle. Etoposide was given intravenously on days 3 and 4 of every cycle. Dose modifications were not made for hematologic or renal toxicity. Chemotherapy was delayed until blood counts or renal function recovered. 


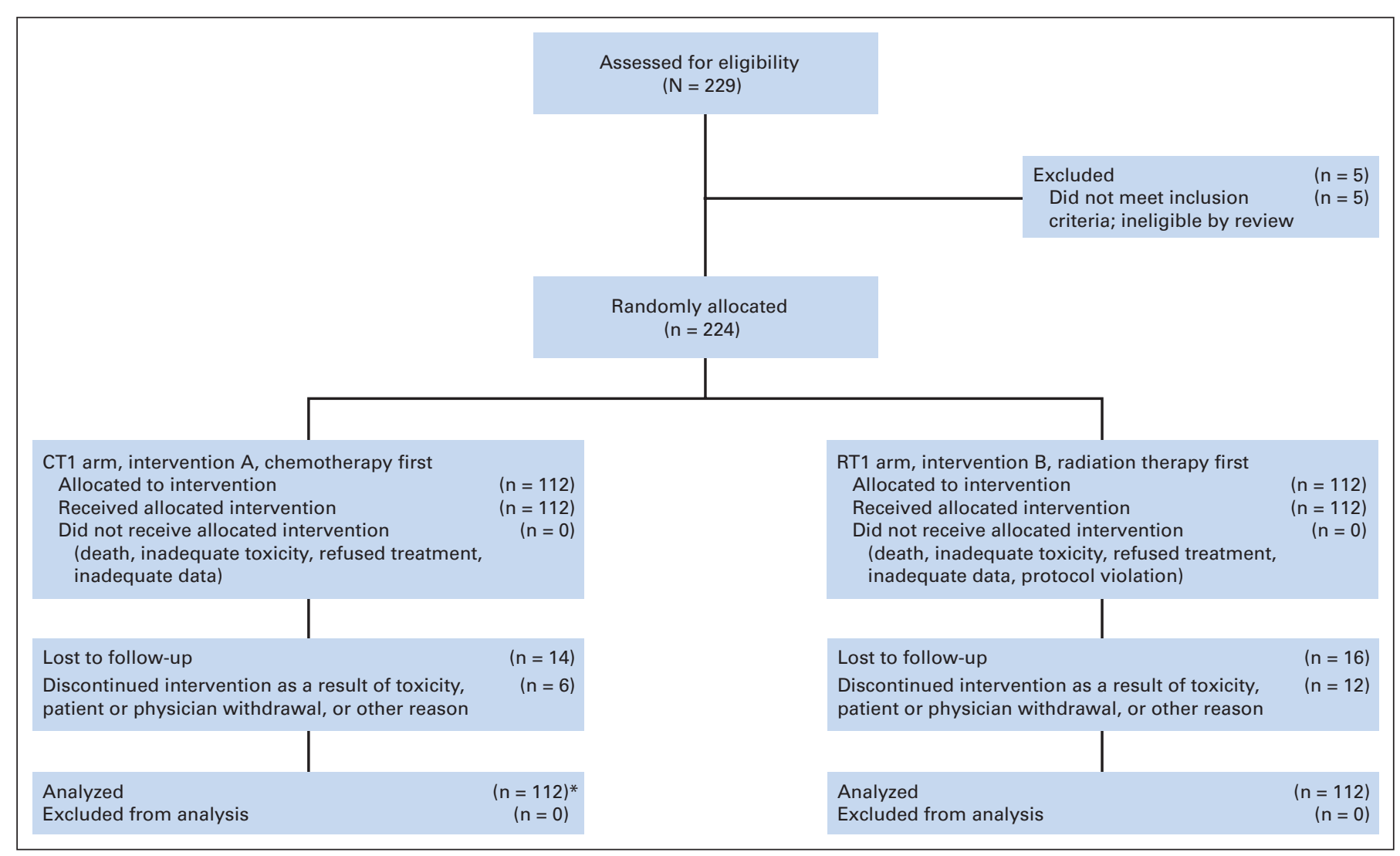

Fig 1. CONSORT diagram. CT1, chemotherapy first; RT1, radiation therapy first. (*) Patients lost to follow-up or off study were censored at date last seen.

The consolidation phase of chemotherapy was given 3 weeks after radiation therapy in the CT1 arm or after blood counts had recovered in the RT1 arm; it consisted of seven cycles of cyclophosphamide $1,000 \mathrm{mg} / \mathrm{m}^{2}$ and vincristine $2.0 \mathrm{mg} / \mathrm{m}^{2}$ administered every 4 weeks. The vincristine was given on day 1 , and the cyclophosphamide was given on days 1 and 2, with mesna 360 $\mathrm{mg} / \mathrm{m}^{2}$ administered to prevent cyclophosphamide-induced hemorrhagic cystitis. No dose modification of maintenance chemotherapy was made for neutropenia, but patients with platelet nadirs of less than $20,000 / \mu \mathrm{L}$ received a $25 \%$ reduction in the cyclophosphamide dose at the next cycle and were supported with platelet transfusions. Trimethoprim-sulfamethoxazole prophylaxis was given during chemotherapy.

\section{Radiation}

Radiation therapy was administered to all patients under treatment. Patients on the CT1 arm received radiation therapy on weeks 10 to 16 after three cycles of cisplatin and etoposide. Patients on the RT1 arm received

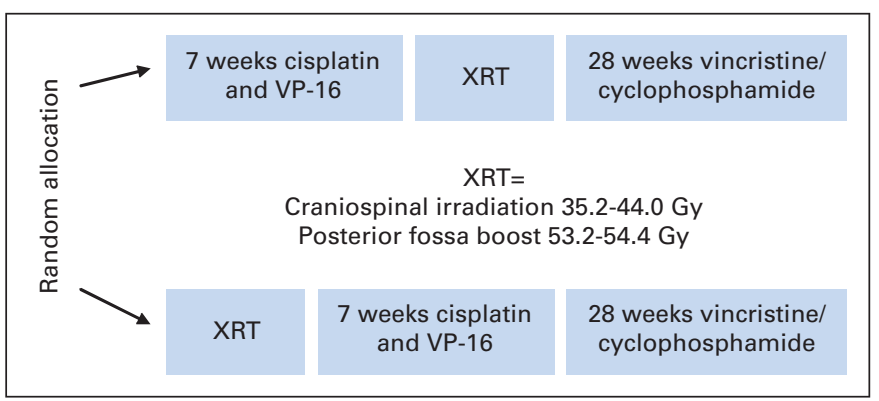

Fig 2. Pediatric Oncology Group 9031 study treatment schematic. VP-16, etoposide; XRT, radiation therapy. radiation therapy on weeks 1 to 7 before any chemotherapy was administered. Details of radiation techniques and quality assurance have been published. ${ }^{14}$

The treatment volume consisted of the whole brain and spinal contents, with a boost to the posterior fossa. Spinal metastases were boosted with individual portals with a margin of one vertebral body on either side of the lesion.

The whole brain and spinal cord for patients with M0-1 disease received $35.2 \mathrm{~Gy}$ in 22 fractions at $1.6 \mathrm{~Gy}$ per fraction prescribed to midplane for the whole brain and to the midthoracic posterior edge of the vertebral body. Patients with M2-3 disease received 40.0 Gy at 1.6 Gy per fraction in 25 fractions to the brain and spinal cord. The posterior fossa boost for patients with M0-1 disease was 18.0 Gy in 10 fractions (1.8 Gy per fraction), for a total of 53.2 Gy to the posterior fossa. In patients with M2-3 disease, the posterior fossa received a boost of $14.4 \mathrm{~Gy}$ in 8 fractions (1.8 Gy per fraction), for a total dose of $54.4 \mathrm{~Gy}$. Spinal or brain metastases were boosted to a total dose of 44.8 Gy at $1.6 \mathrm{~Gy}$ per fraction. Cranial spinal irradiation was delayed if the absolute neutrophil count decreased to less than $750 / \mu \mathrm{L}$ or the platelet count decreased to less than $50,000 / \mu \mathrm{L}$.

\section{Follow-Up Protocol}

On completion of treatment, patients were seen at 2-month intervals until 6 months after treatment, at which time they were observed every 3 months until 2 years after treatment. Thereafter, they were observed every 6 months until 5 years after treatment and then yearly after the 5 -year mark. Imaging examinations were obtained 2 and 6 months after treatment, and then biannually until 5 years after treatment, and then yearly; spinal MRI scans or myelograms were obtained during the first 2 years of follow-up, after which patients were monitored with brain MRI or CT only.

\section{Statistical Considerations}

The primary end point for this study is EFS, defined as the time to disease progression, disease relapse, occurrence of a second neoplasm, or death from 
any cause, measured from the time of study enrollment. The comparison involves all eligible randomly assigned patients. Progression is assessed at any time during or after radiotherapy. This study assumed a 2-year EFS of $60 \% \pm$ $10 \%$ and is sensitive to a $15 \%$ difference with a total enrollment of 220 patients (110 patients to each arm) at $P=.05$ (two-sided) and $80 \%$ power. The end point of overall survival (OS) was defined as time to death from any cause. Nonparametric log-rank tests and Kaplan-Meier curves were used to compare the two groups. Cox regression analysis was used to analyze possible prognostic factors for the risk of recurrence. Response at the end of chemotherapy was compared for the two treatment groups using the $\chi^{2}$ test. Complete response was defined as the disappearance of all radiographically discernible lesions, as well as two consecutively negative CSF cytologic examinations. A partial response was defined as a $50 \%$ or greater reduction in tumor size as measured by the sum of the products of the maximum perpendicular diameters of all measurable lesions or two consecutively negative CSF cytologies in addition to a less than $50 \%$ reduction in tumor size. Stable disease was defined as a less than $50 \%$ reduction in tumor size and a persistently negative or positive CSF cytology. Progressive disease was defined as a more than $25 \%$ increase in the size of any measurable lesion or the appearance of a new lesion.

\section{Quality Control}

The pathology, operative reports, radiology and radiation simulation films, and port films all were reviewed centrally. Details of radiotherapy quality control have been published separately. ${ }^{14}$

\section{RESULTS}

Two hundred twenty-nine patients were enrolled onto the POG 9031 study for high-risk medulloblastoma between July 1990 and March 1996. Five patients found to be ineligible were removed from the study, leaving 224 patients eligible after random assignment. Three of the ineligible patients were misdiagnosed with medulloblastoma, with the other two patients failing to meet high-risk disease criteria. Central review of images was performed at Quality Assurance Review Center. ${ }^{14}$ The CT1 and RT1 arms each accrued 112 eligible patients. The median follow-up time was 6.4 years (range, 0.1 to 11.2 years). The median age was 7.8 years, with a range of 3.0 to 21.4 years. There were 132 males and 92 females, with a variety of races represented ( 34 black, 171 white, 12 Hispanic, and seven Asian patients). The frequency of $\mathrm{M}$ stage was as follows: $\mathrm{M} 0, \mathrm{n}=116$; and $\mathrm{M}+, \mathrm{n}=108$ (M1, $\mathrm{n}=29 ; \mathrm{M} 2$, $\mathrm{n}=36 ; \mathrm{M} 3, \mathrm{n}=34$; and $\mathrm{M} 4, \mathrm{n}=9$ ). Seventy-two patients were $\mathrm{T} 3 \mathrm{~b} / \mathrm{T} 4 \mathrm{M} 0$ with no residual disease (40 patients in the CT1 arm and 32 patients in RT1 arm). Treatment groups were balanced at random assignment with respect to sex, stage, and ethnicity $(P>.05)$. Table 1 lists more detailed patient characteristics in each group.

\section{Outcome by Treatment Group}

Fifty-four patients experienced recurrence or progression, with 43 experiencing subsequent death. Twenty-two patients died of disease without a reported overt progression before this time. Four patients experienced a second neoplasm as a first event. Two patients developed myelodysplastic syndrome, one on each of the treatments, at 3.3 and 2.4 months from study enrollment. Two patients in the RT1 arm had a fibroma and leiomyosarcoma at 5.1 and 5.7 years from study enrollment, respectively. Five-year EFS and OS probabilities for the entire patient cohort as determined by institutional review were $68.1 \% \pm 3 \%$ and $74.6 \% \pm 3 \%$, respectively. Figure 3 shows EFS by randomly assigned treatment. Five-year EFS was $66.0 \% \pm 4.5 \%$ and $70.0 \% \pm 4.4 \%$ in the CT1 and RT1 arms, respectively (log-rank, $P=$ $.54)$. Five-year OS was $73.1 \% \pm 4.2 \%$ and $76.1 \% \pm 4.1 \%$ in the CT1

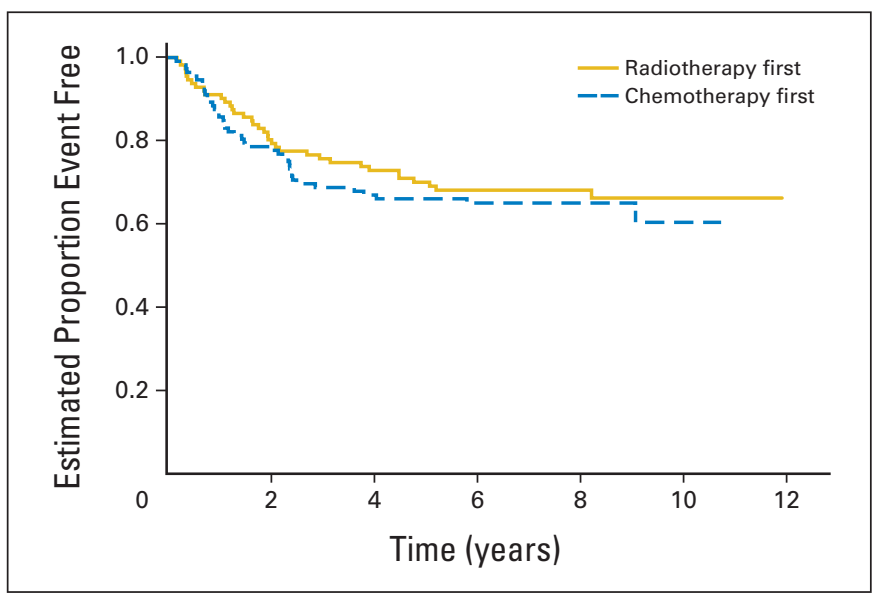

Fig 3. Event-free survival by treatment arm for eligible patients.

and RT1 arms, respectively (log-rank, $P=.47$ ). The log-rank test for detecting differences between both the EFS and OS curves for the two treatments indicate that these curves are not significantly different. There was also no evidence of a difference in EFS between treatment groups after stratifying for stage. In patients with M0 and residual disease after surgery, the 5-year EFS was 59.6\% $\pm 7.0 \%$ in the CT1 arm and $65.1 \% \pm 6.8 \%$ in the RT1 arm (log-rank, $P=.40)$. In patients with $\mathrm{M}+$ and residual disease, the 5 -year EFS was $51.2 \% \pm 9.2 \%$ in the CT1 arm and $64.0 \% \pm 9.3 \%$ in the RT1 arm (log-rank, $P=.20$ ).

\section{Response to Chemotherapy Treatment}

Response was evaluated at the end of 7 weeks of chemotherapy with cisplatin $90 \mathrm{mg} / \mathrm{m}^{2}$ and etoposide $150 \mathrm{mg} / \mathrm{m}^{2}$. Response data for the 224 eligible patients are listed in Table 2. Of the 224 patients, 118 were evaluable for response. One-hundred six patients were not evaluable for response ( 99 patients did not have measurable disease after surgery; one patient on the CT1 arm had an early death on study enrollment; five patients on the RT1 arm had missing response data; and one patient on the RT1 arm was not evaluable for response). The objective response rate (complete response + partial response) was $66 \%$ ( 40 of 61 patients) in the CT1 arm and 86\% (49 of 57 patients) in the RT1 arm $\left(P=.01\right.$, two-sided $\chi^{2}$ test $)$. There was no significant difference in EFS between patients in the CT1 arm who achieved complete or partial response and those who did not (5-year EFS: $73 \% \pm 7 \% v 56 \% \pm 17 \%$, respectively; $P=.1$ ).

\section{Prognostic Factors}

Table 3 lists 5-year EFS and OS within patient categories defined by sex, race, regimen, and $M$ stage. On the basis of a Cox regression

\begin{tabular}{|lrrr|}
\hline \multicolumn{4}{c}{ Table 2. Response Data for Eligible Patients } \\
\hline \multirow{3}{*}{ Response } & No. of Patients \\
\cline { 2 - 4 } & Chemotherapy First & Radiotherapy First & Total \\
\hline Complete response & 19 & 25 & 44 \\
Partial response & 21 & 24 & 45 \\
Stable disease/no response & 9 & 0 & 17 \\
Progressive disease & 12 & 55 & 12 \\
Not evaluable for response & 51 & 112 & 224 \\
Total & 112 & & \\
\hline
\end{tabular}




\begin{tabular}{|c|c|c|c|c|c|c|}
\hline \multirow[b]{2}{*}{ Characteristic } & \multicolumn{3}{|c|}{ 5-Year EFS } & \multicolumn{3}{|c|}{ 5-Year OS } \\
\hline & $\begin{array}{l}\text { Rate } \\
(\%)\end{array}$ & SE (\%) & $P$ & $\begin{array}{l}\text { Rate } \\
(\%)\end{array}$ & SE (\%) & $P$ \\
\hline Sex & & & .96 & & & .90 \\
\hline Male & 68.6 & 4.1 & & 73.7 & 3.9 & \\
\hline Female & 67.3 & 4.9 & & 75.9 & 4.5 & \\
\hline Race & & & .75 & & & .48 \\
\hline White & 69.3 & 3.6 & & 75.7 & 3.3 & \\
\hline Black & 61.0 & 8.5 & & 66.6 & 8.3 & \\
\hline Hispanic & 75.0 & 12.5 & & 82.5 & 11.3 & \\
\hline Other & 57.1 & 18.7 & & 71.4 & 17.1 & \\
\hline Randomly assigned regimen & & & .54 & & & .47 \\
\hline $\mathrm{CT} 1$ & 66.0 & 4.5 & & 73.1 & 4.2 & \\
\hline RT1 & 70.0 & 4.4 & & 76.1 & 4.1 & \\
\hline M stage & & & $<.001^{*}$ & & & $<.001^{*}$ \\
\hline Mo & 74.0 & 4.1 & & 81.6 & 3.6 & \\
\hline M1 & 64.9 & 9.0 & & 68.3 & 8.8 & \\
\hline M2 & 69.2 & 7.7 & & 77.6 & 7.0 & \\
\hline M3 & 61.6 & 8.4 & & 64.5 & 8.3 & \\
\hline M4 & 22.2 & 13.9 & & 33.3 & 15.7 & \\
\hline$M+(M 1, M 2, M 3$, or $M 4)$ & 61.6 & 4.7 & & 67.1 & 4.6 & \\
\hline $\begin{array}{l}\text { Abbreviations: CT1, chemoth } \\
\text { survival; RT1, radiation therap } \\
\text { "Log-rank } P \text { value in compari }\end{array}$ & $\begin{array}{l}\text { erapy } \\
\text { y first. } \\
\text { ing } M\end{array}$ & $\begin{array}{l}\text { first; EF } \\
4 v \text { othe }\end{array}$ & $\begin{array}{l}\text { event-f } \\
\text { (MO, N }\end{array}$ & $\begin{array}{l}\text { free sur } \\
11, \mathrm{M} 2\end{array}$ & $\begin{array}{l}\text { rvival; OS } \\
\text { or M3). }\end{array}$ & S, overall \\
\hline
\end{tabular}

analysis, factors such as sex, race, and regimen were not statistically significant predictors of EFS or OS, and M stage was the only factor associated with outcome. The 5-year EFS rates for M4 patients and patients with M0, M1, M2, or M3 disease were $22 \% \pm 14 \%$ and $70 \% \pm 3 \%$, respectively $(P<.001)$. The relative risk for progression or relapse was four times (95\% CI, 1.9 to 8.9 times) higher for M4 patients than for those with M0, M1, M2, or M3 disease. The relative risk for death was 4.3 times (95\% CI, 2.0 to 9.4 times) higher for M4 patients than for those with M0, M1, M2, or M3 disease.

\section{Site of First Relapse}

The site of first relapse was similar for the two treatments arms. The most common site of first relapse was the posterior fossa $(n=10)$, and the second most common site was the supratentorium $(\mathrm{n}=9$; Table 4). Approximately one third of the relapses were in the posterior fossa. The median time to relapse was 6.36 years (range, 0.16 to 11.93 years). In the CT1 arm, the median time to relapse was 6.22 years (range, 0.16 to 10.75 years), whereas in the RT1 arm, it was 6.68 years (range, 0.18 to 11.93 years). However, follow-up protocols only included spinal MRI within the first 2 years after diagnosis. Subsequent follow-up imaging was by CT or MRI of the brain. This may have skewed the initial site of relapse to intracranial and under-reported the incidence of spinal failures.

\section{Radiation Therapy}

Patients with M0-1 disease received a median of 35.2 Gy CSI and 53.2 Gy to the posterior fossa. Patients with M2-3 disease received a median of 40 Gy CSI and 54.4 Gy to the posterior fossa. This did not differ by treatment group. The average overall radiation therapy treatment times in the CT1 and RT1 arms were 46.3 days (standard deviation, 10.51 days) and 44.8 days (standard deviation, 8.98 days), respectively. Twenty-two patients in CT1 group and 11 patients in RT1 group had more than 50 treatment days. This comparison had a $P=.06$ based on the Fisher's exact test.

\section{Toxicity}

Regarding acute toxicity, there were more episodes of thrombocytopenia in the RT1 arm, but otherwise, the toxicity for the two treatments was similar. Treatment-induced diseases include two patients with myelodysplastic syndrome and a third patient who developed an ossifying fibroma.

\section{DISCUSSION}

The OS and EFS rates in this study, both for all high-risk patients, as defined in 1990, and for those with metastatic disease within the CNS (M1-3), are comparable to data previously reported in this disease setting. ${ }^{4,5,8,9,15}$ There was no difference in EFS or OS between immediate or delayed radiation therapy. The EFS rates observed in this study were better than the $60 \%$ nominal rate at year 2 , assumed in our power calculations. However, the observed rate difference between study arms was small, only $4 \%$ and $3 \%$ for EFS and OS, respectively, at year 5. Our lack of treatment effect contrasts with the results of the German randomized controlled trial of patients with both low- and high-risk medulloblastoma, in which the RT1 group did significantly better than the CT1 group. ${ }^{9}$ Note that in the German study, patients in the CT1 arm, which included M0-3 stages, had a prolonged radiation therapy treatment time because of greater hematologic toxicity than the patients in the RT1 arm. This is important because prolonged treatment time has been associated with a decrease in EFS. ${ }^{8,16-19}$

\begin{tabular}{|c|c|c|c|c|c|c|c|c|c|}
\hline \multirow[b]{3}{*}{ Site } & \multicolumn{9}{|c|}{ No. of Patients } \\
\hline & \multicolumn{4}{|c|}{ Chemotherapy First } & \multicolumn{4}{|c|}{ Radiation Therapy First } & \multirow[t]{2}{*}{ Total } \\
\hline & Total & Mo & M1-2 & M3 & Total & Mo & M1-2 & M3 & \\
\hline Supratentorial (brain, cerebral hemispheres, corpus callosum) & 5 & 3 & 2 & 0 & 4 & 3 & 1 & 0 & 9 \\
\hline Posterior fossa (cerebellum, cerebellar peduncles) & 5 & 3 & 0 & 2 & 5 & 3 & 2 & 0 & 10 \\
\hline CSF/meninges/dural sinus/ventricular system & 5 & 1 & 2 & 2 & 2 & 0 & 0 & 2 & 7 \\
\hline Spinal cord & 1 & 1 & 0 & 0 & 1 & 0 & 1 & 0 & 2 \\
\hline Bone & 0 & 0 & 0 & 0 & 3 & 2 & 0 & 1 & 3 \\
\hline Total & 16 & 8 & 4 & 4 & 15 & 8 & 4 & 3 & 31 \\
\hline
\end{tabular}


There was no difference in EFS between the treatment arms for patients with residual disease after surgery either in the posterior fossa or elsewhere. Also, in contrast to the findings in other studies, the presence of residual disease in the posterior fossa was not associated with worse EFS for patients with M0 disease. Patients with M0 disease and no significant residual disease after resection of their T3b or T4 lesion had EFS of approximately $73 \% \pm 5.2 \%$. Note that in the current era of medulloblastoma treatment, these patients would be categorized as being standard risk.

The EFS and OS of patients with M0 and M1 disease compare favorably with other randomized studies ${ }^{4,5,7,18,20}$ and are consistent with their findings. However, the 5-year EFS for the patients with M2-3 disease was higher than in previously published reports (EFS of $>60 \%$ compared with $30 \%$ in the German study). ${ }^{9}$ On the basis of previously published relatively poor survival rates, some patients with M2-3 disease have been assigned directly to high-dose chemotherapy with stem-cell rescue regimens. ${ }^{15,21}$

It is important to note that the high EFS rates in the M2-3 patients may be the result of a higher dose of radiation used. In these patients, $40 \mathrm{~Gy}$ to the whole brain and spinal cord were delivered with a boost of 4.8 Gy to the gross disease. The EFS rates are similar to a multiinstitutional phase II study demonstrating a 67\% PFS in patients with $\mathrm{M}+$ disease in which 11 of the 15 patients had either M2 or M3 disease. A similar chemotherapy regimen was used consisting of lo- mustine, cisplatin, and vincristine. In this study, patients received 36 Gy to the craniospinal axis with a boost to 45 Gy to areas of gross disease outside of the posterior fossa. ${ }^{7}$ This approach in high-risk patients may be a reasonable alternative to high-dose chemotherapy with stem-cell rescue in the older child. However, the late effects of increased radiation will need to be studied in long-term survivors.

The EFS and OS reported in this study compare favorably with other trials examining high-risk medulloblastoma. Preirradiation chemotherapy fails to confer a survival advantage over radiation before chemotherapy.

\section{AUTHORS' DISCLOSURES OF POTENTIAL CONFLICTS OF INTEREST}

The author(s) indicated no potential conflicts of interest.

\section{AUTHOR CONTRIBUTIONS}

Conception and design: Nancy J. Tarbell, Henry Friedman, Larry Kun

Provision of study materials or patients: Nancy J. Tarbell

Collection and assembly of data: Peter Burger, Patrick Barnes

Data analysis and interpretation: Nancy J. Tarbell, William R.

Polkinghorn, Torunn Yock, Tianni Zhou, Zhengjia Chen, Larry Kun

Manuscript writing: All authors

Final approval of manuscript: All authors

\section{REFERENCES}

1. Gurney JG, Davis $S$, Severson RK, et al: Trends in cancer incidence among children in the U.S. Cancer 78:532-541, 1996

2. McNeil DE, Coté TR, Clegg $L$, et al: Incidence and trends in pediatric malignancies medulloblastoma/ primitive neuroectodermal tumor: A SEER updateSurveillance Epidemiology and End Results. Med Pediatr Oncol 39:190-194, 2002

3. Polkinghorn WR, Tarbell NJ: Medulloblastoma: Tumorigenesis, current clinical paradigm, and efforts to improve risk stratification. Nat Clin Pract Oncol 4:295-304, 2007

4. Evans AE, Jenkin RD, Sposto R, et al: The treatment of medulloblastoma: Results of a prospective randomized trial of radiation therapy with and without CCNU, vincristine, and prednisone. J Neurosurg 72:572-582, 1990

5. Tait DM, Thornton-Jones $\mathrm{H}$, Bloom HJ, et al: Adjuvant chemotherapy for medulloblastoma: The first multi-centre control trial of the International Society of Paediatric Oncology (SIOP I). Eur J Cancer 26:464-469, 1990

6. Packer RJ, Sutton LN, Elterman R, et al: Outcome for children with medulloblastoma treated with radiation and cisplatin, $\mathrm{CCNU}$, and vincristine chemotherapy. J Neurosurg 81:690-698, 1994

7. Bailey CC, Gnekow A, Wellek S, et al: Prospective randomised trial of chemotherapy given before radiotherapy in childhood medulloblastoma: International Society of Paediatric Oncology (SIOP) and the (German) Society of Paediatric Oncology (GPO)—SIOP II. Med Pediatr Oncol 25:166-178, 1995
8. Zeltzer PM, Boyett JM, Finlay JL, et al: Metastasis stage, adjuvant treatment, and residual tumor are prognostic factors for medulloblastoma in children: Conclusions from the Children's Cancer Group 921 randomized phase III study. J Clin Oncol 17:832-845, 1999

9. Kortmann RD, Kühl J, Timmermann B, et al: Postoperative neoadjuvant chemotherapy before radiotherapy as compared to immediate radiotherapy followed by maintenance chemotherapy in the treatment of medulloblastoma in childhood: Results of the German prospective randomized trial HIT ' 91 . Int J Radiat Oncol Biol Phys 46:269-279, 2000

10. Taylor RE, Bailey $C C$, Robinson $K J$, et al: Outcome for patients with metastatic (M2-3) medulloblastoma treated with SIOP/UKCCSG PNET-3 chemotherapy. Eur J Cancer 41:727-734, 2005

11. Gajjar $A$, Chintagumpala $M$, Ashley $D$, et al: Risk-adapted craniospinal radiotherapy followed by high-dose chemotherapy and stem-cell rescue in children with newly diagnosed medulloblastoma (St Jude Medulloblastoma-96): Long-term results from a prospective, multicentre trial. Lancet Oncol 7:813-820, 2006

12. Packer RJ, Goldwein J, Nicholson HS, et al: Treatment of children with medulloblastomas with reduced-dose craniospinal radiation therapy and adjuvant chemotherapy: A Children's Cancer Group study. J Clin Oncol 17:2127-2136, 1999

13. Packer RJ, Gajjar A, Vezina G, et al: Phase III study of craniospinal radiation therapy followed by adjuvant chemotherapy for newly diagnosed averagerisk medulloblastoma. J Clin Oncol 24:4202-4208, 2006

14. Miralbell R, Fitzgerald TJ, Laurie $F$, et al: Radiotherapy in pediatric medulloblastoma: Quality assessment of Pediatric Oncology Group Trial 9031. Int J Radiat Oncol Biol Phys 64:1325-1330, 2006

15. Strother D, Ashley D, Kellie SJ, et al: Feasibility of four consecutive high-dose chemotherapy cycles with stem-cell rescue for patients with newly diagnosed medulloblastoma or supratentorial primitive neuroectodermal tumor after craniospinal radiotherapy: Results of a collaborative study. J Clin Oncol 19:2696-2704, 2001

16. Chan AW, Tarbell NJ, Black PM, et al: Adult medulloblastoma: Prognostic factors and patterns of relapse. Neurosurgery 47:623-631, 2000

17. Miralbell R, Bleher A, Huguenin $P$, et al: Pediatric medulloblastoma: Radiation treatment technique and patterns of failure. Int J Radiat Oncol Biol Phys 37:523-529, 1997

18. Thomas PR, Deutsch M, Kepner JL, et al: Lowstage medulloblastoma: Final analysis of trial comparing standard-dose with reduced-dose neuraxis irradiation. J Clin Oncol 18:3004-3011, 2000

19. del Charco JO, Bolek TW, McCollough WM, et al: Medulloblastoma: Time-dose relationship based on a 30-year review. Int J Radiat Oncol Biol Phys 42:147-154, 1998

20. Taylor RE, Bailey CC, Robinson $K$, et al: Results of a randomized study of preradiation chemotherapy versus radiotherapy alone for nonmetastatic medulloblastoma: The International Society of Paediatric Oncology/United Kingdom Children's Cancer Study Group PNET-3 Study. J Clin Oncol 21:15811591, 2003

21. Papadakis V, Dunkel IJ, Cramer LD, et al: High-dose carmustine, thiotepa and etoposide followed by autologous bone marrow rescue for the treatment of high risk central nervous system tumors. Bone Marrow Transplant 26:153-160, 2000 
Tarbell et al

\section{Acknowledgment}

We thank James Kepner, $\mathrm{PhD}$, for statistical support throughout this trial, and Chris Williams-Hughes for her work in finalizing the manuscript. 